\title{
NOTAS
}

\section{En Vitoria: En la Imprènta nueva de Doña Manuela de Eqquerra, Impressora de esta M. N. y M. L. Provincia de Alava, [1762-1763?]}

\author{
María Eugenia LÓPEZ VAREA
}

(Biblioteca de la Universidad Pontificia Comillas)

\section{Resumen}

Descubrimiento de un nuevo impreso de Manuela de Ezquerra, quien regentó su propia imprenta en Vitoria, en el siglo XVIII, mientras estaba casada con Bartolomé de Riesgo, primer impresor de Vitoria y de Álava. Se recoge su producción conocida hasta el momento, así como el último impreso descubierto en la Biblioteca de la Universidad Pontificia Comillas de Madrid.

Palabras clave: Imprenta. Mujeres impresoras. Manuela de Ezquerra. Bartolomé de Riesgo. Vitoria. Álava. Siglo XVIII. Universidad Pontificia Comillas. Biblioteca. Patrimonio Bibliográfico.

\section{En Vitoria: En la Imprènta nueva de Doña Manuela de Eqquerra, Impressora de esta M. N. y M. L. Provincia de Alava, [1762-1763?]}

Abstract

Discovery of a new printed of the Manuela de Ezquerra, who directed her own printing press in Vitoria, in the XVIII Century, when she married to Bartolomé de Riesgo, first printing of Vitoria and Alava. Her known production is collected, including the last printed discovered in the Library of the Universidad Pontificia Comillas de Madrid. 
Keywords: Printing. Women printers. Manuela de Ezquerra. Bartolomé de Riesgo. Vitoria. Alava. XVIII Century. Universidad Pontificia Comillas. Library. Bibliographic Heritage.

A Pilar Izuzquiza Rueda.

\section{Introducción}

Al estudiar el establecimiento de la imprenta en Álava, comprobamos que no se conocía ningún impreso editado en ella hasta que, en la primavera del año 1722, Bartolomé de Riesgo instaló su taller en la calle Correría n 110 de Vitoria, del que salieron, al menos, dos obras diferentes bajo su marca ese mismo año, según las investigaciones de Julio-César Santoyo y Santiago Asenjo ${ }^{1}$.

En la biografía de Riesgo elaborada por $S_{a n t o y o}{ }^{2}$, nos encontramos con la figura de su esposa, Manuela de Ezquerro, de la que aporta datos biográficos referidos fundamentalmente a partidas de bautismo, tanto de ella como de sus hijos, así como escritos administrativos relacionados con las instituciones de Logroño, San Sebastián, Álava o Vitoria. Es en esta última documentación donde aparece, sin explicación sobre cuándo ocurrió, el cambio de apellido de Manuela -de Ezquerro a Ezquerra-, lo que no parece que pueda interpretarse como producto fortuito de una errata, ya que se halla en varias partes en el mismo escrito y, además, fue la propia Manuela la que adoptó la forma Ezquerra para el pie de imprenta de los impresos salidos de su taller, por lo que parece probable que este cambio se hubiera producido antes.

Finalmente, se ha elaborado una relación con las obras editadas por Manuela de Ezquerra - con datos de ejemplares-, entre las que se incluye un impreso desconocido hasta ahora y que figura en la misma con el número 5: Razon de los lugares de las diez y ocho hermandades, que necesitan despachos de el Señor Diputàdo Generál (Fig. 1). Dicho impreso ha sido encontrado formando parte de las guardas de una colección de cuatro libros de Derecho civil, que recoge comentarios al Digesto de Justiniano en una edición latina de Lyon de 1569 - en la que no figura el nombre de la imprenta-, perteneciente al patrimonio

\footnotetext{
1 Julio-César SANTOYO, Santiago ASENJO, «Una primicia desconocida de la imprenta alavesa: LaVida de santa Coleta de fray Damián Cornejo (1722)», Sancho el Sabio, 13 (2000), pp. 211-226.

2 Julio-César SANTOYO, La imprenta en Álava: Historia, obras, documentos. Vol. I. El Siglo XVIII, Vitoria-Gasteiz, Fundación Sancho el Sabio, 1995.
}

Titivillus, ISSN 2387-0915, ISSN-e 2603-9966, 2 (2016), pp. 199-210 
bibliográfico de la Biblioteca de la Universidad Pontificia Comillas de Madrid, con signaturas DC-60 (3 volúmenes) y DC-58 (1 volumen).

\section{Bartolomé de Riesgo y de los Monteros de Espinosa y Manuela de Ezquerro y Sáenz de Chávarri.}

La biografía de Bartolomé de Riesgo y Montero, consultadas las obras de Serdán ${ }^{3}$ y Santoyo ${ }^{4}$, así como las más recientes de Manzanos-Vives ${ }^{5}$ y Díez de Uré-Roda $^{6}$ —estas dos últimas basadas en la mencionada de Santoyo, sin aportar ninguna novedad-, vemos que ha sido estudiada con mayor profundidad por Julio-César Santoyo, quien proporciona referencias sobre documentación de archivo y ejemplares impresos por este impresor conservados en bibliotecas, por lo que resulta obligado realizar constantes citas a su obra a lo largo de este artículo. Así, Santoyo indica que Bartolomé de Riesgo debió nacer a finales del siglo XVII en Madrid y que, en enero de 1721, contrajo matrimonio en Logroño con Manuela de Ezquerro y Sáenz de Chávarri, a la sazón viuda del impresor Diego Revilla Mendoza, quien a su vez provenía de una familia de impresores zaragozanos y era propietario de un taller en Logroño, donde Riesgo habría ejercido de oficial.

Por su parte, Manuela de Ezquerro, nacida en Los Arcos (Navarra) en 16877, pertenecía a una familia de impresores instalados en Pamplona desde 1704 -donde desarrollarán su actividad hasta comienzos del siglo XIX-, por lo que conocía y había desempeñado muchas de las tareas que se llevan a cabo en una imprenta cuando se casó con el impresor Diego Revilla, establecido en Logroño, y con el que tuvo dos hijas nacidas en esta ciudad, -María Fermina, en julio de 1718, y Manuela, en marzo de 1720-. Al poco tiempo de quedarse viuda, y como era frecuente entre los impresores ${ }^{8}$, contrajo matrimonio con el oficial Bartolomé de Riesgo, con el que tendría cuatro hijos - María Javiera, nacida en Logroño el 30 de noviembre de 1721, y Josefa Cristina, Lorenzo

\footnotetext{
3 Eulogio Serdán y Aguirregavidia, Rincones de la Historia Vitoriana..., Vitoria, Imprenta Provincial, 1922.

${ }^{4}$ SANTOYO, La imprenta en Álava...

5 Paloma Manzanos Arreal, Francisca Vives Casas, Las mujeres en Vitoria-Gasteiz a lo largo de los siglos: Recorridos y biografias, Vitoria-Gasteiz, Ayuntamiento, 2001.

6 Ana Díez de Uré ERAul, Paco Roda Hernández, Tierra de estrELLAS: Guía histórica de las mujeres de Tierra Estella, Tafalla (Navarra), Altaffaylla, 2004.

7 Víctor PASTOR ABÁIgAR, «Juan Joseph Ezquerro, hijos y nieto, una familia de impresores», Principe de Viana, 247 (2009), pp. 441-493.

${ }^{8}$ Fermín de los ReYes GÓMEZ, El libro en España y América: Legislación y censura (Siglos XVXVIII), Madrid, Arco/Libros, 2000, 2 vols.; José Eugenio de EgUIZÁBAL, Apuntes para una bistoria de la legislación española sobre imprenta desde el año 1480 al presente, Facsímil de la ed. de 1879, Pamplona, Analecta, 2003.
} 
José y María Josefa Dorotea, nacidos los tres en Vitoria, en 1723, 1725 y 1727, respectivamente-

Bartolomé de Riesgo había conseguido el nombramiento de impresor oficial de Logroño justo antes de trasladarse con Manuela de Ezquerro y sus tres primeras hijas a Vitoria, donde instaló imprenta y librería, logrando la vecindad en esta ciudad el 13 de marzo de 1722 y pasando a ser el primer impresor de la misma. Poco tiempo después, Riesgo presentó al Ayuntamiento la solicitud para ser nombrado impresor oficial de Vitoria, lo que obtuvo el 5 de septiembre de $1722^{\circ}$, por lo que, a partir de ese momento, firmará sus trabajos como «Impressor de la Ciudad» o como «Impresor de la Muy Noble y Muy Leal Ciudad».

El 20 de noviembre, presentó una nueva solicitud, esta vez a las Juntas Generales de Álava, para ser nombrado impresor de la Provincia, lo que también le fue concedido. A partir de entonces, en su pie de imprenta suelen aparecer ambos reconocimientos: «Impressor de dicha Provincia, y de la Ciudad», «Provinciae \& urbis Typographum», «Impressor de la M.N. Provincia de Alava y de dicha Ciudad», «Impressor de las Muy Nobles y Muy Leales Provincia de Alava y Ciudad de Vitoria», etc.

Uno de sus primeros trabajos que ha llegado hasta nosotros coincide con el que, hasta el año 2000, figuraba como primera publicación impresa en Vitoria y, a su vez, en Álava: el Quaderno de Leyes y Ordenanzas con que se govierna esta Muy Noble y Muy Leal Provincia de Alava y diferentes privilegios, y Cedulas de su Magestad $^{10}$. Pero, tras los últimos descubrimientos bibliográficos de Santoyo y Asenjo, el Quaderno compartirá su condición de primer impreso de Álava con La vida de santa Coleta, del franciscano Damián Cornejo, ya que ambos presentan el mismo pie de imprenta: «En Vitoria: Por Bartholomè Riesgo, Impressor de la Ciudad. Año 1722» ${ }^{11}$. Al figurar en ambos casos únicamente el título de «Impressor de la Ciudad», se puede deducir que habrían sido impresos entre el 5 de septiembre y el 20 de noviembre de 1722, pero no sabemos cuál lo fue primero o si se hicieron simultáneamente. Sólo hay constancia de que el Quaderno estaba ya impreso el 13 de octubre de $1722^{12}$ y que, a diferencia de éste, que era un encargo oficial, la Vida de santa Coleta se hizo «a devocion de una Devota».

De los quince años que Riesgo permaneció en Vitoria como impresor han llegado hasta nosotros una cincuentena de obras salidas de su taller, aunque es muy probable que editara bastantes más, pero la tipología de muchas de ellas ha podido favorecer su desaparición.

\footnotetext{
${ }^{9}$ SANTOYO, La imprenta en Álava...

${ }^{10}$ Ibídem.

${ }^{11}$ SANTOYO, ASENJO, «Una primicia...».

${ }^{12}$ Ibídem.
}

Titivillus, ISSN 2387-0915, ISSN-e 2603-9966, 2 (2016), pp. 199-210 
En 1738, Riesgo dejó al frente de la imprenta de Vitoria a su yerno Tomás de Robles y Navarro, casado con su hijastra Manuela Revilla Ezquerro el año anterior, ya que había decidido trasladarse a San Sebastián, ciudad que estaba sin impresor desde 1734 y de la que había obtenido el encargo de imprimir en 1735, en su taller de Vitoria, las actas de las Juntas Generales de la Provincia de Guipúzcoa, tras lo cual recibió el ofrecimiento de ser impresor oficial de dicha provincia, lo que aceptó, instalándose en San Sebastián ese mismo año, «con parte de su familia, su Imprenta y Muebles» ${ }^{13}$.

Riesgo estuvo trabajando en ambos talleres a la vez, desde 1735 hasta 1738, en que abandonó el de Vitoria definitivamente tras dejar a su yerno Tomás de Robles al frente del mismo, quien lo regentará hasta su muerte en 1781. Durante los más de cuarenta años que permaneció en la imprenta de Vitoria, Robles editó más de mil obras, según Santoyo ${ }^{14}$, aunque apenas nos ha llegado la mitad de ellas. En su taller, sólo usó papel francés, de los molinos transpirenaicos, - de I. Cassou, fabricado en Lanne (Bearn); de Jules Fargues, elaborado en Saint Jean Pied de Port; de J. Ferran, hecho en Soues (Bigorre); o de M.I. Monie realizado en Bigorre-. Le sucedió su hijo Gregorio Marcos de Robles y Revilla quien desarrolló su labor de impresor de Vitoria desde 1782 hasta 1800, aunque había comenzado a trabajar con su padre desde los doce años. Gregorio Marcos murió soltero, dejando como heredero al frente de la imprenta a su cuñado Fermín Articarena de Larumbe, viudo de Juana de Robles. Al morir Larumbe, le sucedió su viuda, Micaela de Soloeta y Alcíbar, y a ésta los hijos que tuvo Larumbe con su primera mujer, Juana de Robles, que continuaron al frente de la imprenta hasta 1866, en que morirá soltero el último descendiente de la saga familiar.

La documentación de archivo señala que Bartolomé de Riesgo se instaló en San Sebastián en 1738, con su mujer Manuela de Ezquerro y parte de sus hijos, y estuvo al frente de la imprenta donostiarra hasta 1751, en que pasa el testigo a su hijo Lorenzo José de Riesgo, ya que, según las fuentes consultadas por Santoyo, Bartolomé de Riesgo aparece, en 1768, en la ciudad de Caracas, como evaluador testamentario del Jefe de Escuadra José de Iturriaga ${ }^{15}$, probablemente por mantener negocios con la Compañía Guipuzcoana de Caracas, de la que había sido impresor oficial mientras estuvo viviendo en San Sebastián. Es posible que en Venezuela se dedicara al contrabando de libros con ideas ilustradas, según Arántzazu Amézaga ${ }^{16}$, y parece ser que poseía una imprenta ambulante en la que habría impreso octavillas.

\footnotetext{
13 Ibídem.

${ }^{14}$ SANTOYO, La imprenta en Álava...

15 SANTOYO, ASENJO, «Una primicia...».

16 Arántzazu Amézaga Iribarren, «La Real Compañia Guipurcoana de Caracas. Crónica sentimental con una visión historiográfica. Los años áuricos y las rebeliones (1728-1751)», Sancho el Sabio, 23 (2005), pp. 167-208.
} 
Manuela de Ezquerro, como hija, esposa y madre de impresores conocía el oficio, ya que habría tenido que participar activamente en tareas que, aunque apenas estén documentadas - como preparación de tintas, limpieza de tipos, cosido de cuadernillos, etc.-, eran necesarias para el buen funcionamiento del negocio ${ }^{17}$.

Cada vez que Bartolomé de Riesgo se trasladaba a otra ciudad en donde establecía su imprenta, Manuela seguía desempeñando su trabajo en ella, además de encargarse de sus hijos.

Manuela había ido con su marido de Logroño a Vitoria y luego a San Sebastián, donde permaneció cuando Bartolomé de Riesgo comenzó su aventura americana.

No se sabe con seguridad desde qué año se encuentra Riesgo en Venezuela, pero todo parece indicar que es anterior a 1762, fecha en la que Manuela y el hijo de ambos, Lorenzo José de Riesgo, figuran como vecinos de Vitoria y al frente de una imprenta en esta ciudad, aunque Lorenzo José seguiría regentando la imprenta de San Sebastián hasta 1802, en que se trasladó a Galicia.

\section{De Manuela de Ezquerro a Manuela de Ezquerra impresora}

Manuela de Ezquerro no acompañó a su marido a Venezuela, sino que se quedó en San Sebastián, al frente de la familia y ayudando a su hijo Lorenzo José en el negocio hasta que, pasados los años, regresó a Vitoria, donde su hijo estableció una imprenta para ella, como recoge Santoyo del Memorial redactado por Lorenzo José donde asegura que «la deja con una copiosa, y lucida oficina, y Oficiales del mayòr desempeño... $»^{18}$. Este Memorial iba dirigido a la Diputación, dado que ya existía una imprenta en Vitoria, la del yerno de Manuela, Tomás de Robles, quien recibía todos los encargos oficiales. Por ello y para que su madre pudiera mantenerse recibiendo también dichos encargos, Lorenzo José había propuesto en el Memorial destinado a la Diputación que ambas imprentas se alternasen en la tarea de editar los documentos oficiales, figurando él como «Impressor de esta Provincia», aunque fuera su madre la que regentara realmente la imprenta de Vitoria.

17 Rosa María CAPEL MarTínez (coord.), Mujer y sociedad en España (1700-1975), Madrid, Instituto de la Mujer, 1986; Lourdes GUTIÉRREZ, Purificación LAFUENTE, Laura CARrILlo, Mujeres Impresoras: Guía de recursos bibliográficos, Madrid, BNE, Servicio de Información Bibliográfica, Disponible en: <http://www.bne.es/es/Micrositios/Guias/ MujeresImpresoras / > [consulta: 12 de octubre de 2015]; Albert CORBETO LÓPEZ, «Notas para el estudio de las impresoras españolas (siglos XVI-XVIII)», Más que viudas y huérfanas. Mesa: Impresoras, editoras y diseñadoras en la historia, Centro Cultural España de México, 16 de abril de 2008, < http://bg.biograficas.com/secciones/docs_complementarios/Corbeto.pdf> [consulta: 12 de octubre de 2015].

18 SANTOYO, La imprenta en Alava...

Titivillus, ISSN 2387-0915, ISSN-e 2603-9966, 2 (2016), pp. 199-210 
Ante esto, Tomás de Robles presentó un Contramemorial, el 24 de noviembre de 1762, durante las Juntas Generales de Santa Catalina, donde, tras debatirse y votarse «por mayoría de Votos, se resolvió, que la Impression de los Decrétos, y demás Cosas de ésta M. N. y M. L. Provincia ... siga al cuidado, y Cargo de Doña Manuela de Ezquèrra [sic], y Tomàs Robles, su Yerno, alternando, para el efecto, entre sí por años.... ${ }^{19}$, el primero para Manuela de Ezquerra - que a partir de este momento figurará con el apellido terminado en «a», no sólo en el Memorial, tal como aparece en la documentación de archivo, sino también en el pie de imprenta de los trabajos realizados en su taller-y el siguiente año para Tomás de Robles.

La solicitud, el Memorial y todos los trámites administrativos son realizados por Lorenzo José, quien incluso se nombra «Impressor de esta Provincia». Pero, a partir de la aprobación de las Juntas Generales de Santa Catalina en noviembre de 1762, el nombre de Manuela de Ezquerra aparece ya en los impresos oficiales de Vitoria hasta finales de 1763, sin ninguna referencia masculina de la que dependiera: «Doña Manuela de Ezquerra, Impressora de esta M. N. y M. L. Provincia de Alava» $\mathrm{O}$ «En la Imprènta nueva de Doña Manuela de Ezquerra, Impressora de esta M. N. y M. L. Provincia de Alava».

El cambio de apellido de Manuela, de Ezquerro a Ezquerra, no parece que sea producto de una errata administrativa ocurrida a raíz del Memorial dirigido por su hijo Lorenzo a la Diputación de Vitoria, sino que debió haber ocurrido antes, ya que se mantiene varias veces a lo largo del Memorial, así como en el Contramemorial de su yerno, y, como puede comprobarse en la relación que figura a continuación, es la propia Manuela la que lo incluirá con normalidad en todos los impresos salidos de su imprenta, lo que nos lleva a pensar que dicho cambio se habría producido con anterioridad, aunque, por ahora, no tenemos constancia de cuándo se llevó a cabo por primera vez.

Por el acuerdo de las Juntas Generales, Manuela de Ezquerra regentó la imprenta de Vitoria de 1762 a 1763 con autonomía, pero como sus impresos no suelen tener fecha en el pie de imprenta tampoco se puede asegurar si alguno es posterior, aunque, a partir de este último año de 1763, ya no hay constancia de más trabajos salidos de su taller $\multimap$ estos no han llegado hasta nosotros-, debido principalmente a que la concesión oficial pasaba durante el siguiente año a Tomás de Robles, lo que influiría en una menor producción del taller de Manuela de Ezquerra quien deberá dedicarse a conseguir encargos privados. Quizá la falta de trabajo pudo ocasionar que la imprenta cerrara, pero sólo es una suposición que habría que intentar comprobar con documentación de archivo, si existiera. Tampoco sabemos si Manuela de Ezquerra permaneció en Vitoria o si retornó a San Sebastián, lo que parece ser que hizo, al menos, al final de sus días.

Las últimas noticias que tenemos sobre Manuela de Ezquerra aparecen en un escrito que realizó su marido desde Venezuela el 15 de marzo de 1768,

\footnotetext{
19 Ídem.
} 
donde Bartolomé de Riesgo se declaraba «residente en Caracas y vecino de San Sebastián, capital de la Provincia de Guipúzcoa», como recoge María Dolores Fernández de Casadevante ${ }^{20}$, tras lo cual otorgaba un poder al Alcalde de esta ciudad, con motivo del fallecimiento de su mujer Manuela de Ezquerra en San Sebastián sin hacer testamento. Con el poder otorgado solicitaba un inventario de bienes para que estos pasasen a su hijo Lorenzo José, a quien nombró heredero universal en su testamento, firmado el 30 de marzo de 1770 en Caracas.

\section{Impresos de Manuela de Ezquerra}

No sabemos con seguridad cuántos impresos salieron de la imprenta de Manuela de Ezquerra, y es posible que muchos hayan desaparecido. No obstante, en ocasiones hay descubrimientos fortuitos que contribuyen a profundizar en la historia y el conocimiento de la imprenta, como ocurre con el impreso número 5 de la siguiente relación.

Al establecer el catálogo de los impresos conocidos hasta ahora elaborados en el taller de Manuela de Ezquerra, parece conveniente comenzar por los recogidos por Julio-César Santoyo ${ }^{21}$ :

1.-

Decretos celebrados por esta M. N. y M. L. provincia de Alava, en sus Juntas Generales de Santa Cathalina, año de 1762, siendo Diputado General, el Señor D. Pedro Ortiz de Zarate y Guebara... . - En Vitoria: Por Doña Manuela de Ezquèrra, Impressóra de ésta Muy Noble, y Muy Leal Provincia de Alava, [s.a.].

[2], 60 p., [1] h. de grab.

Sign.: [1, A2-P2

Impreso, muy probablemente, en los primeros meses de 1763, en papel fabricado en Soues, Bigorre, por la viuda de Jean Monie.

Precede al título: +

La h. de grab. calc. fue grabada en Madrid en 1671 y representa a San Prudencio y el escudo de Álava.

Ejemplares:

ARCHIVO DEL TERRITORIO HISTÓRICO DE ÁLAVA, Vitoria: AC-I-3.

BiblioteCA, Fundación SANCHO El SABIO, Vitoria: Es el ejemplar que ha sido digitalizado.

Impreso digitalizado: Obra original perteneciente a los fondos bibliográficos de la Fundación Sancho el Sabio Fundazioa (Vitoria-Gasteiz)22:

${ }^{20}$ María Dolores Fernández de Casadevante Romaní, «Introducción a la historia de la imprenta en Guipúzcoa (1585-1850)», Revista General de Información y Documentación, 22 (2012), pp. 67-92.

${ }^{21}$ SANTOYO, La imprenta en Alava... 
http://hdl.handle.net/10357/39891

\section{2.-}

Decretos hechos por esta M. N. y M. L. provincia de Alava, en sus Juntas Generales Ordinarias de el mes de mayo del año de 1763, en el lugar de Foronda, siendo Diputado General el señor D. Pedro Ortiz de Zarate y Guebara... . - En Vitoria: por Doña Manuela de Ezquerra, Impressòra de èsta M. N. y M. L. Provincia de Alava, [s.a].

[2], 39 p., [1] p. en bl., [1] h. de grab.

Sign.: A2-I2, K3. Jardel.

La impresión se hizo en 1763, en papel fabricado en Perigord por B.

Precede al título: +

La h. de grab. calc. fue grabada en Madrid en 1671 y representa a San Prudencio y el escudo de Álava.

CCPB000201989-2

Ejemplares:

Biblioteca Seminario Diocesano, Facultad de Teología, Vitoria (VI-SD): PV-V-2/48, ejemplar falto de últimas páginas. Encuadernación en holandesa. Encuadernado junto a otros textos legales, formando un volumen facticio. Es el ejemplar que ha sido digitalizado.

Impreso digitalizado: Obra original perteneciente a los fondos bibliográficos de la Fundación Sancho el Sabio Fundazioa (Vitoria-Gasteiz)23:

http://hdl.handle.net/10357/209

\section{3.-}

Cuenta General de los gastos ordinarios, y extraordinarios de esta Muy Noble y Muy Leal Provincia de Alava, desde el dia veinte y cinco de noviembre de 1761, hasta otro tal del presente de $1762 \ldots$

$35 \mathrm{p}$. 1763.

Carece de pie de imprenta. - Impreso probablemente a comienzos de

Ejemplares:

VI-SD.

A estos tres impresos, hay que agregar los que figuran en el Catálogo Colectivo de Patrimonio Bibliográfico Español, $\mathrm{CCPB}^{24}$, que son, además del

\footnotetext{
22 Fundación SANCho el SABIo Fundazioa, Centro de Documentación de la Cultura Vasca, Vitoria-Gasteiz, Fundación Sancho El Sabio, <http://www.fsancho-sabio.es/> [consulta: 12 de octubre de 2015].

23 Ídem.
}

Titivillus, ISSN 2387-0915, ISSN-e 2603-9966, 2 (2016), pp. 199-210 
que aparece como número 2 tanto en la relación de Santoyo como en la nuestra, el siguiente - del que constan dos ejemplares, uno de ellos en la Iglesia de San Juan Bautista de Laguardia (Álava), del cual el archivero-bibliotecario de la misma, D. Antonio Mijangos, con absoluta amabilidad, nos ha proporcionado para su estudio una copia digitalizada de dicho ejemplar que se encuentra en perfecto estado de conservación y al que agradecemos su valiosa colaboración-:

\section{4.-}

Missa in festo Sancti Prudentij Episcopi Turiasonensis et Patroni principalis Provinciae Alabensis. - En Vitoria : en la Imprènta nueva de Doña Manuela de Ezquerra,[s.a.]

3 p., [1] p. en bl. ; Fol.

Sign.: [12

Precede al tít.: + Die XXVIII. Aprilis.

El Decretum está fechado en 1762.

Datos de impresión tomados del colofón.

Texto a dos columnas y a dos tintas.

Inicial ornada y viñeta final son grab. xil.

ССРВ001084193-8

Ejemplares:

IGLESIA DE SAN JUAN BAUTISTA, LAGUARDIA: A-4-15(10), ejemplar en perfecto estado de conservación. Encuadernación en piel sobre tabla con hierros dorados y deteriorada. Encuadernado junto a otras obras, formando un volumen facticio.

VI-SD: L-10034(2), ejemplar deteriorado afectando al colofón. Encuadernación en pasta deteriorada. Encuadernado junto a otras obras, formando un volumen facticio.

Finalmente, a estos cuatro impresos diferentes añadiremos como primicia el que hemos tenido la suerte de encontrar entre las guardas de varios libros del fondo patrimonial de la Biblioteca de la Universidad Pontificia Comillas de Madrid $^{25}$ - a la que agradecemos las facilidades dadas para su consulta y reproducción (Fig. 1)-, que ha resultado ser un impreso desconocido hasta ahora y cuya descripción es la siguiente:

${ }^{24}$ Catálogo Colectivo de Patrimonio Bibliográfico, Madrid, Ministerio de Cultura, $\mathrm{CCPB}, \quad<$ http://www.mcu.es/bibliotecas/MC/CCPB/index.html> [consulta: 12 de octubre de 2015].

25 Biblioteca de la Universidad Pontificia Comillas, Catálogo en línea, Madrid, Universidad Pontificia Comillas, <http://www.upcomillas.es> [consulta: 12 de octubre de 2015].

Titivillus, ISSN 2387-0915, ISSN-e 2603-9966, 2 (2016), pp. 199-210 
5.-

Razon de los lugares de las diezy ocho hermandades, que necesitan despachos de el Señor Diputàdo Generál, los que se ponen por el Abecedario siguiente ... . - En Vitoria: en la Imprènta nueva de Doña Manuela de Ezquerra, Impressora de esta Muy Noble, y Muy Leal Provincia de Alava, [s.a.]

$1 \mathrm{~h}$.

Sign.: $\square 1$

Sin fecha de impresión.

Precede al título: $](+)[$

Portada enmarcada por orla tipográfica.

Ejemplares:

BiblioteCA DE la UnIVERSIDAD PONTIFICIA COMILlas: DC-58; DC60-1; DC-60-2 y DC-60-3, en todos los casos como hojas de guarda.

\section{Conclusión}

Este último impreso (Fig. 1) descrito con el número 5, que se encontró formando parte de las guardas de la encuadernación de cuatro volúmenes de una colección de Derecho civil con comentarios al Digesto de Justiniano, impresa en latín, y editada en Lyon en 1569 - sin datos de impresor-, se repite en los dos rectos del pliego doblado en folio, con filigrana francesa - se adivina la palabra «Burean»- con escudo. Tanto Tomás de Robles, como Bartolomé de Riesgo, usaron preferentemente papeles fabricados en Francia, algo que Manuela de Ezquerra mantuvo en sus trabajos.

La orla tipográfica de este último impreso coincide con la del impreso editado por Bartolomé de Riesgo en Vitoria en 1730: «Seminario victoriense ... su autor D. Joan Joseph Saenz de Texada ... $»^{26}$, lo que podría llevar a suponer que la imprenta que Lorenzo José de Riesgo estableció para su madre en Vitoria constaría de tipos y materiales antiguos provenientes de la primera época de Bartolomé de Riesgo, habiéndose quedado Lorenzo José con los más modernos para la oficina de San Sebastián.

Consultados los catálogos y repertorios bibliográficos en papel: Palau ${ }^{27}$, Salváa ${ }^{28}$ y Aguilar Piñal ${ }^{29}$, así como las bases de datos y catálogos bibliográficos

\footnotetext{
${ }^{26}$ SANTOYO, La imprenta en Álava...

27 Antonio Palau y DULCET, Manual del librero hispanoamericano. Bibliografía general española desde la invención de la imprenta hasta nuestros tiempos con el valor comercial de los impresos descritos, $2^{\mathrm{a}}$ ed. rev. y añadida por Agustín Palau Claveras, Barcelona-Oxford, Palau, 1948-1977. 38 vols.

28 Pedro Salvá y MallÉN, Catálogo de la biblioteca de Salvá, enriquecido con la descripción de otras muchas obras, de sus ediciones, etc., Valencia, Imprenta de Ferrer de Orga, 1872, 2 vols.

${ }^{29}$ Francisco AgUILAR PIÑAL, Bibliografía de autores españoles del siglo XVIII, Madrid, Consejo Superior de Investigaciones Científicas, CSIC, Instituto Miguel de Cervantes, 1981, 10 vol.
} 
en línea: Catálogo Colectivo de Patrimonio Bibliográfico Español, CCPB30; Catálogo de la Red de Bibliotecas Universitarias de España, REBIUN31; Catálogo de la Biblioteca Nacional de España, BNE ${ }^{32}$; y Catálogo Virtual de la Universidad de Kalsruhe, Kalsruher Virtueller Katalog, KVK ${ }^{33}$, no hemos encontrado más impresos de Manuela de Ezquerra que los ya mencionados, ni tenemos constancia de la existencia de ejemplares, incluido el último impreso, el número 5 , en otras bibliotecas.

Por tanto, estamos ante un impreso desconocido hasta ahora, que se incluye en la producción de Manuela de Ezquerra, una mujer impresora en el Antiguo Régimen, en pleno siglo XVIII —-documentada su actividad desde 1762 a 1763, aunque pudo haberse extendido algo más en el tiempo-, que estuvo al frente de un taller y que con su formación, su trabajo y su determinación contribuyó, con pie de imprenta propio, al desarrollo y a la historia de la imprenta en Vitoria y Álava.

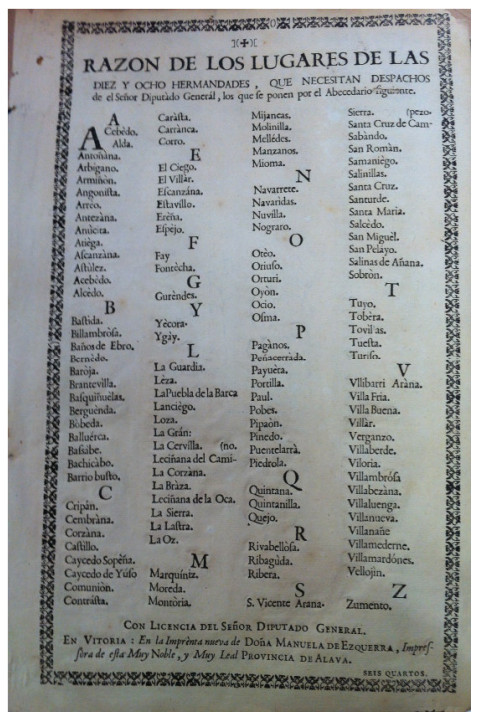

Fig. 1. Impreso desconocido hasta ahora de Manuela de Ezquerra. Biblioteca de la Universidad Pontificia Comillas.

30 CATÁLOGO COLECTIVO DE PATRIMONIO BIBLIOGRÁFICO...

31 Red de Bibliotecas Universitarias de España, Catálogo Colectivo, Madrid, CRUE, REBIUN, <http://www.rebiun.org/catalogoColectivo/Paginas/default.aspx> [consulta: 12 de octubre de 2015].

32 Biblioteca Nacional de España, Catálogo en línea, Madrid, BNE, < http://catalogo. bne.es/uhtbin/webcat> [consulta: 12 de octubre de 2015].

33 KALSRUHER VirTUELLER KATALOG, Kalsruher, KVK, <http://www.ubka.uni-karlsru he.de/kvk_en.html> [consulta: 12 de octubre de 2015].

Titivillus, ISSN 2387-0915, ISSN-e 2603-9966, 2 (2016), pp. 199-210 